\title{
UN SONETO DE SHAKESPEARE DESDE LA TEORÍA DE LA VALORACIÓN
}

\author{
Inés Elena Regueira \\ Universidad de Buenos Aires \\ inesregueira@gmail.com
}

Resumen: En el marco de la Lingüística Sistémico Funcional, la Teoría de la Valoración se utilizará para el análisis de los sentimientos comunicados por un soneto a la dama oscura de Shakespeare. Esta herramienta resulta apropiada porque estudia, precisamente, la representación de los sentimientos en el discurso. El propósito del análisis será indagar en los significados interpersonales del soneto elegido y ver cómo se negocian actitudes y sentimientos, siguiendo la propuesta de Martin y Rose (2003). Se estudiarán los recursos apreciativos, cómo se asignan los valores y se alinea al lector de acuerdo a ellos. También se analizarán los recursos que amplifican, gradúan o suavizan actitudes, las fuentes que las proyectan y la modalidad; todos estos elementos conforman la prosodia del texto.

Palabras clave: Shakespeare, Soneto, Teoría de la Valoración.

\begin{abstract}
Within the framework of Systemic Functional Linguistics, the Theory of Appraisal will be used to analyze the feelings communicated by one of Shakespeare's sonnets to the dark lady. Appraisal Theory is the most appropriate because it studies the representation of feelings in discourse. The purpose of this paper is to look into the interpersonal meanings of the chosen sonnet and observe how attitudes are negotiated, following Martin and Rose (2003). The appreciative resources used in the poem will be analyzed to see how values are assigned and readers aligned with them. Also, the way in which attitudes are emphasized, softened or graded will be studied, together with the sources that project them and modality, all of which create the prosody of the text.
\end{abstract}

Keywords: Shakespeare, Sonnet, Appraisal Theory. 


\section{Introducción}

Un poema es también un acto de discurso que pone en práctica un saber discursivo para relacionarse con los demás y comunicar sentimientos, juicios o valoraciones. Entendiendo al texto como una unidad semántica (Halliday, 1994), trataremos de comprender la relación entre los significados interpersonales en uno de los sonetos a la dama oscura y las elecciones que realizó Shakespeare para construir su texto y comunicar sus juicios y valoraciones a sus contemporáneos y a nosotros.

Dentro de una clasificación de la poesía por sus formas, siguiendo a Jitrik, en los "sonetos, villancicos, odas, baladas se privilegia la organización que pone en evidencia cierto orden de sabiduría productiva" (Jitrik, 2008, p. 55). El soneto, entonces, tiene una forma rígida de estructura. Sin embargo, Shakespeare logra construir significados que evaden la forma esperada para ese contexto cultural y esa forma poética.

Los sonetos como forma poética desarrollan una argumentación que invita al lector a acompañarla hasta la conclusión. La estrategia que usa Shakespeare en los sonetos a la dama oscura consiste en contradecir con el pareado final el principio argumentativo implícito que le permitió llegar a la conclusión en el tercer cuarteto, lo cual produce una reacción de sorpresa en el lector. Vemos aquí un ejemplo del recurso de extrañamiento indicado por Shklovsky en Art as Technique (Harrison et al., 2003, p. 280), este extrañamiento está presente en la ruptura con lo que esperamos del soneto pues la 
conclusión que se encuentra en el pareado final no procede de la línea argumentativa de los cuartetos. Como también dice el formalista ruso, una nueva forma aparece no para expresar un contenido nuevo, sino para reemplazar una forma antigua (Harrison et al., 2003, p. 277).

Creemos que este extrañamiento en los sonetos a la dama oscura no es una cuestión meramente formal, sino que es parte de las opciones que elige el autor para representar las relaciones interpersonales y construir su texto. La Teoría de la Valoración (Appraisal) constituye una herramienta que nos provee la Lingüística Sistémico Funcional para este tipo de análisis y por ello se introducirá brevemente el marco de la teoría que utilizan Martin y Rose en su libro Working with Discourse (2003).

La naturaleza interactiva del discurso está presente en las actitudes que se negocian en un texto, o sea, sus significados interpersonales. La Teoría de la Valoración se ocupa de identificar estos significados y los recursos apreciativos que los constituyen. La evaluación de las actitudes negociadas en un texto incluye la fuerza de los sentimientos involucrados, la forma en que los valores son asignados y cómo los lectores se alinean de acuerdo con esos valores.

Los significados interpersonales no se realizan solamente en forma local dentro de una fase de un texto, sino que se acumulan a lo largo de este y dan un "color" al discurso que forma la "prosodia de la actitud" (Martin y Rose, 2003, p. 27). En esta acumulación de significados, los lectores son alineados retóricamente mientras el texto se desarrolla. La Teoría de la 
Valoración (Appraisal) se ocupa de analizar los significados interpersonales e identificar los recursos que establecen el tono de un texto, así como de reconocer las elecciones que se presentan en distintos momentos del discurso. Este esquema de elecciones conforma la "prosodia" del texto y construye la voz del que "aprecia" y de la comunidad con la que comparte valores.

Hay diferentes clases de actitudes, entre ellas las que expresan sentimientos y pueden ser entonces positivas o negativas, directas o implícitas. Este es el caso del poeta que confiesa en el Soneto 130 "I love to hear her speak" [Amo escuchar a la dama hablar], expresando así un sentimiento positivo explícito.

También hay actitudes que emiten juicios positivos o negativos, implícitos o explícitos sobre las personas, sus cualidades personales o calidad moral. Un ejemplo de un juicio negativo implícito se observa en el Soneto 134 cuando el poeta se define como "I my self am mortgaged to thy will" [Yo mismo estoy hipotecado a la voluntad de la amada].

Y, por último, hay actitudes que evalúan objetos y productos o seres humanos vistos como entidades. Esta apreciación puede ser positiva o negativa y conforma, junto con los sentimientos y las evaluaciones de las personas, un marco de actitudes que corresponde a un contexto de situación y de cultura en el cual se negocian esas actitudes. En el caso del Soneto 130, las diferentes partes del cuerpo de la dama son evaluadas negativamente para el contexto de cultura de la época, que privilegia una belleza pálida y rubia. 
Las actitudes son amplificadas con diferentes recursos discursivos, entre ellos los intensificadores, los indicadores de cantidad, manera y modo, el uso de léxico emotivo, el uso de metáforas y la elección de elementos léxicos o gramaticales que agregan gradación a las actitudes expresadas en la prosodia de los sentimientos de un texto. En los sonetos los ejemplos de amplificación de actitudes abundan, ya sea por el uso de comparativos, como en el caso del Soneto 130, "Coral is far more red than her lips' red" [el coral es más rojo que sus labios], o por el léxico elegido, como se puede ver en el Soneto 135 cuando el poeta describe su actitud hacia la dama con el verbo "vex" [fastidiar].

También se considera que las actitudes son agudizadas o suavizadas cuando se gradúa aquello que no es inherentemente graduable, cuando se precisa una cantidad o se desdibuja una cualidad. En el Soneto 135, el poeta se pregunta si lo que para otros "seem right gracious" [parece correcto], en él no es aceptado. Todos estos recursos delinean actitudes que construyen los significados interpersonales del texto.

El abordaje de Martin y Rose tiene en cuenta, a su vez, las fuentes de donde provienen las actitudes, es decir, dónde se originan las evaluaciones que conforman la prosodia textual. El discurso referido, las citas, se consideran proyecciones de las fuentes que originaron el discurso, y constituyen al texto como heteroglósico. La proyección está presente en los actos de habla, en la relación entre las oraciones o dentro de la cláusula, $y$ en expresiones o frases entre comillas que aluden a otro enunciador. Los sonetos a la dama oscura presentan una notable heteroglosia porque el origen de muchos de sus juicios 
y valoraciones está en fuentes externas al diálogo entre los amantes: proviene de la sociedad a la que pertenecen, de su contexto de situación.

Otra forma de incluir voces en el discurso es a través de la modalidad, la cual sirve a dos propósitos de negociación solicitar servicios o información- que se mueven en escalas de positivo a negativo. En esta escala, la polaridad negativa invoca dos voces: la que habla y la que se opone y es negada en el texto. Una de las tantas instancias de esta forma de inclusión de la heteroglosia se puede observar claramente en el soneto 149, cuyas líneas introductorias negocian información utilizando la polaridad negativa, e incluyendo así la voz de la dama que se niega a reconocer que el poeta la ama: "Canst thou, O cruel, say I love thee not, / When I against myself with thee partake?", [¿Puedes tú, mujer cruel, decir que no te amo, cuando yo participo contigo en contra de mí mismo?]. La negación proclama una voz en relación a otra potencial enunciación que opone su punto de vista; la negación, entonces, siempre implica la presencia de dos voces.

La modalidad abre un espacio de negociación dentro de cinco tipos: regularidad, probabilidad, obligación, inclinación y habilidad. Al igual que la polaridad, los recursos de modalización presentan un espacio de negociación en el cual se reconocen voces alternativas que dialogan acerca de una sugerencia o reclamo. La fuerza de la enunciación varía de acuerdo al verbo modal que plantea la negociación. Así, se abre también una escala que va desde la obligación hasta la posibilidad. Entre los muchos ejemplos de esta forma de inclusión de otras voces en el texto, el soneto 143 en su 
pareado final (línea 13) utiliza la variante de probabilidad que atenúa la fuerza del ruego: "So will I pray that thou mayst have thy will" [Entonces rogaré para que puedas obtener lo que deseas]. Claramente, así se hacen presentes en esta línea las voces del poeta y de su amada.

La concesión es otro recurso que indica heteroglosia y agrega un elemento de contra-expectativa. Cuando el poeta concede que su amor es inusual para ese contexto (Soneto 130), pero aun así lo defiende, está respondiendo sorpresivamente a la argumentación que desarrolla el soneto y que detalla los defectos de la dama.

En resumen, la prosodia del texto se desenvuelve a lo largo de este y surge de las elecciones sobre el tono y modo en que estas se despliegan a lo largo del discurso. Son estas elecciones las que construyen la voz del que evalúa los sentimientos, conductas y actitudes y definen así posturas en forma dinámica. El sistema de valoración se constituye por la selección de una o varias voces que están presentes a través de las proyecciones, la modalidad o la concesión, por la evaluación de sentimientos, conductas u objetos y por la gradación que permite amplificar, agudizar o suavizar actitudes.

El propósito de indagar en los significados interpersonales nos permite, según White:

Explorar cómo se construyen los textos, no sólo para persuadir explícitamente sino también para influir y, en última instancia, en la naturalización de actitudes, creencias y supuestos, de manera más indirecta, más implícita. (White, 2004, p. 4). 
En los sonetos 127 a 154 de Shakespeare, la dama aparece en principio definida como oscura, negra, y sus atributos son en general negativos tomando en consideración su contexto de producción y de cultura (siglo XVI). Sus labios no son rojos, sus cabellos son alambres, su aliento no se parece a un perfume ni camina como una diosa. Estas características están amplificadas con intensificadores como "nothing like the sun" [nada parecido al sol], "no such roses see I in her cheeks" [no hay tales rosas en sus mejillas] o "music hath a far more pleasing sound" [la música tiene un sonido mucho más bello que su voz].

Los sentimientos que el poeta expresa hacia la dama son de amor, pero un amor que lo atormenta, un amor cruel. La expresión de este amor es directa en los sonetos y está amplificada por adjetivación e intensificadores: "my dear doting heart" [mi querido y doliente corazón], "tis my heart that loves what they despise, / Who, in despite of view, is pleased to dote" [mi corazón se complace en amar a pesar de lo que ven mis ojos].

A esta construcción hay que agregar también el uso de recursos como la metáfora. Los ojos que "Be anchored in the bay where all men ride" [están anclados en una bahía donde todos los hombres navegan] construyen parte de la expresión de celos y de alusión al carácter promiscuo de la amada.

El efecto acumulativo de los recursos usados para expresar los sentimientos del poeta, la "prosodia" del texto, nos relata una historia de sentimientos controvertidos, que alternan lo 
positivo y lo negativo de una relación entre dos personas, que a su vez está atravesada por otros que intervienen con sus voces.

Estas voces presentes en el texto emiten juicios morales acerca del carácter de la dama y del poeta. En algunos versos aparece la mención a lo que "ellos dicen" y lo que el poeta "jura" reconocer en su amada. Esta proyección de las fuentes del juicio también aparece en actos de habla como en el Soneto 131: "Thy black is fairest in my judgment's place" [en mi juicio tu negro es lo más bello], o en cláusulas en el mismo soneto: "do witness bear" [los testigos prestan testimonio]. Los sonetos, entonces, son textos heteroglósicos en los que una comunidad dentro de un determinado contexto cultural, emite juicios sobre la conducta moral a seguir. La dama oscura no respeta esas convenciones y, aunque el poeta las reconoce como auténticas y adhiere a ellas, las contradice al amar a la dama.

\section{Ejemplo de análisis desde la Teoría de la Valoración}

Soneto 130

\begin{tabular}{|l|l|l|}
\hline Soneto 130 & Actitud & $\begin{array}{l}\text { Atribución. } \\
\text { Origen }\end{array}$ \\
\hline $\begin{array}{l}\text { My mistress' eyes are } \\
\text { nothing like the sun; } \\
\text { [Los ojos de mi amada } \\
\text { no son en absoluto } \\
\text { como el sol] }\end{array}$ & Apreciación negativa. & $\begin{array}{l}\text { Heteroglosia } \\
\text { (negativo). } \\
\text { Ojos como el } \\
\text { sol: } \\
\text { comparación } \\
\text { cliché de la }\end{array}$ \\
\hline
\end{tabular}




\begin{tabular}{|c|c|c|}
\hline & & $\begin{array}{l}\text { poesía } \\
\text { medieval. }\end{array}$ \\
\hline $\begin{array}{l}\text { Coral is far more red than } \\
\text { her lips are red } \\
\text { [El coral es mucho más rojo } \\
\text { que sus labios] }\end{array}$ & $\begin{array}{l}\text { Apreciación negativa. } \\
\text { Amplificación. }\end{array}$ & $\begin{array}{l}\text { Comparación } \\
\text { del coral con } \\
\text { los labios: } \\
\text { cliché. } \\
\text { Introduce la } \\
\text { voz de los } \\
\text { poemas de } \\
\text { amor. }\end{array}$ \\
\hline $\begin{array}{l}\text { If snow be white, why } \\
\text { then her breasts are dun } \\
\text { [Si la nieve es blanca, } \\
\text { entonces sus pechos } \\
\text { son oscuros] }\end{array}$ & $\begin{array}{l}\text { Apreciación negativa. } \\
\text { Léxico emotivo, dun: } \\
\text { pardo. }\end{array}$ & $\begin{array}{l}\text { Heteroglosia } \\
\text { (lenguaje } \\
\text { referido). } \\
\text { Pechos } \\
\text { blancos } \\
\text { como nieve: } \\
\text { cliché. }\end{array}$ \\
\hline $\begin{array}{l}\text { If hairs be wires, black } \\
\text { wires grow on her head } \\
\text { [Si los cabellos son } \\
\text { cables, negros cables } \\
\text { crecen en su cabeza] }\end{array}$ & $\begin{array}{l}\text { Apreciación negativa. } \\
\text { Léxico emotivo, } \\
\text { wires: cables. }\end{array}$ & $\begin{array}{l}\text { Heteroglosia } \\
\text { (lenguaje } \\
\text { referido). }\end{array}$ \\
\hline $\begin{array}{l}\text { I have seen roses } \\
\text { damasked, red } \\
\text { white } \\
\\
{[\mathrm{He} \text { visto }}\end{array}$ & Apreciación positiva. & $\begin{array}{l}\text { Heteroglosia } \\
\text { (acto de } \\
\text { habla). }\end{array}$ \\
\hline
\end{tabular}




\begin{tabular}{|c|c|c|}
\hline $\begin{array}{l}\text { aterciopeladas, rojas y } \\
\text { blancas] }\end{array}$ & & \\
\hline $\begin{array}{l}\text { But no such roses see I in } \\
\text { her cheeks } \\
\text { [Pero no veo tales rosas } \\
\text { en sus mejillas] }\end{array}$ & $\begin{array}{l}\text { Apreciación negativa. } \\
\text { Amplificación. }\end{array}$ & $\begin{array}{l}\text { Mejillas } \\
\text { como rosas: } \\
\text { cliché. }\end{array}$ \\
\hline $\begin{array}{l}\text { And in some perfumes } \\
\text { there is more delight } \\
\text { [Y en algunos perfumes } \\
\text { hay más placer] }\end{array}$ & $\begin{array}{l}\text { Apreciación positiva. } \\
\text { Podría ser Afecto } \\
\text { positivo: la reacción } \\
\text { que producen los } \\
\text { perfumes es de } \\
\text { placer. } \\
\text { Amplificación. }\end{array}$ & $\begin{array}{l}\text { Heteroglosia } \\
\text { (lenguaje } \\
\text { referido). }\end{array}$ \\
\hline $\begin{array}{l}\text { That in the breadth that } \\
\text { from my mistress reeks } \\
\text { [Que en el olor del } \\
\text { aliento de mi señora] }\end{array}$ & $\begin{array}{l}\text { Apreciación negativa. } \\
\text { Léxico emotivo, reek: } \\
\text { emanar mal olor. }\end{array}$ & $\begin{array}{l}\text { Perfume en } \\
\text { el aliento: } \\
\text { cliché. }\end{array}$ \\
\hline $\begin{array}{l}\text { I love to hear her } \\
\text { speak, yet well I know } \\
\text { [Amo escucharla } \\
\text { hablar, aunque sé } \\
\text { perfectamente] }\end{array}$ & $\begin{array}{l}\text { Afecto positivo: } \\
\text { proceso mental de } \\
\text { reacción. } \\
\text { Juicio positivo. }\end{array}$ & $\begin{array}{l}\text { Heteroglosia } \\
\text { (acto de } \\
\text { habla). }\end{array}$ \\
\hline $\begin{array}{l}\text { That music bath a far } \\
\text { more pleasing sound } \\
\text { [Que la música tiene un }\end{array}$ & $\begin{array}{l}\text { Apreciación positiva. } \\
\text { Afecto positivo: } \\
\text { proceso mental de }\end{array}$ & \begin{tabular}{ll}
\multicolumn{2}{c}{ Comparación } \\
entre & la \\
música y la
\end{tabular} \\
\hline
\end{tabular}




\begin{tabular}{|c|c|c|}
\hline $\begin{array}{l}\text { sonido mucho más } \\
\text { placentero] }\end{array}$ & $\begin{array}{l}\text { reacción. } \\
\text { Amplificación. }\end{array}$ & $\begin{array}{l}\text { voz de la } \\
\text { amada: } \\
\text { cliché. }\end{array}$ \\
\hline $\begin{array}{l}\text { I grant I never saw a } \\
\text { goddess go } \\
\text { [Aseguro que jamás vi a } \\
\text { una diosa caminar] }\end{array}$ & & $\begin{array}{l}\text { Heteroglosia } \\
\text { (acto de } \\
\text { habla y } \\
\text { negación). }\end{array}$ \\
\hline $\begin{array}{l}\text { My mistress when she } \\
\text { walks treads on the } \\
\text { ground } \\
{[\mathrm{Mi} \text { dama cuando }} \\
\text { camina pisa el suelo] }\end{array}$ & $\begin{array}{l}\text { Apreciación negativa. } \\
\text { Léxico emotivo, } \\
\text { tread: pisotear. }\end{array}$ & \\
\hline $\begin{array}{l}\text { And yet, by heaven, I } \\
\text { think my love as rare } \\
\text { [Y aún, Dios mío, creo } \\
\text { que mi amor es tan } \\
\text { extraordinario] }\end{array}$ & $\begin{array}{l}\text { Apreciación positiva. } \\
\text { Léxico, rare: único. } \\
\text { Afecto positivo: } \\
\text { reacción de sorpresa. } \\
\text { Léxico, by heaven: } \\
\text { interjección. }\end{array}$ & $\begin{array}{l}\text { Heteroglosia, } \\
\text { acto de } \\
\text { habla, } \\
\text { concesión. }\end{array}$ \\
\hline $\begin{array}{l}\text { As any she belied with } \\
\text { false compare } \\
\text { [Como cualquiera con } \\
\text { el que ella presuma } \\
\text { comparar] }\end{array}$ & $\begin{array}{l}\text { Apreciación positiva. } \\
\text { Léxico, belie: falsear. }\end{array}$ & $\begin{array}{l}\text { Heteroglosia, } \\
\text { discurso } \\
\text { referido. }\end{array}$ \\
\hline
\end{tabular}

Consignas: 
Texto subrayado: discurso atribuido a otras voces

Texto en cursiva: actitud implícita

Texto en azul: juicio moral

Texto en verde: apreciación de valor

Texto en rojo: expresión de sentimientos

La prosodia del texto distribuye significados interpersonales de apreciación negativa hacia los rasgos de la amada en un contexto de cultura que describe a las damas como blancas, luminosas, perfumadas, con cabellos rubios y mejillas rosadas. La concesión del pareado final introduce otra voz que disiente con ese contexto y valora positivamente su amor contradiciendo el juicio de su época. En este contexto de situación, en el que el caballero elogia a su amada, el enunciador se opone al discurso oficial. En esta parte de la relación amorosa, el yo lírico admira lo que los demás rechazan, elige fuera de su contexto de situación y de cultura.

El uso de intensificadores como "nothing like the sun"[no se parece en nada al sol] o "far more red" [mucho más rojo] manifiestan una voluntad de exacerbar los rasgos negativos en oposición a lo esperable en el contexto de cultura. Las oraciones condicionales que introducen las líneas 3 y 4 ("If snow be White" [Si la nieve es blanca] / "If hairs be wires" [Si los cabellos son cables]) dan lugar a la voz de ese contexto que promulga cómo deben ser los pechos y el cabello de las damas que merecen ser amadas. A partir de la novena línea la prosodia se transforma lentamente en apreciación positiva 
hacia los rasgos de la amada y culmina abiertamente con una defensa de su amor frente a otros amores falsos.

Stephen Booth, en su edición comentada de los Sonetos, aclara que la tradición del amor cortés consideraba "fair" [hermosa] a la dama rubia de ojos azules, pero que también existía la contra-tradición que alababa los ojos y cabellos negros, y menciona como ejemplos a Tasso y Sidney (Booth, 1977, p. 434).

El propósito comunicativo del autor es presentar las características de la dama como negativas y así obtener la adhesión del lector que comparte la misma cultura y valores de belleza, para luego sorprenderlo con su afirmación de que, a pesar de todo, su amor no puede ser comparado con ningún otro.

\section{Evaluación}

El análisis de los significados interpersonales muestra especialmente un diálogo entre el poeta y su comunidad de pertenencia, diálogo que plantea posiciones encontradas y pone en evidencia la rebeldía del poeta frente a las convenciones de su contexto de cultura. Para responder a las voces de la moral de la época se apela a apreciaciones, juicios y sentimientos que logran alinear al lector con el poeta y en contra de las convenciones.

Desde el punto de vista de la Lingüística Sistémico Funcional, los textos son heteroglósicos porque otras voces 
son aludidas de diferentes maneras. En el soneto analizado, la mención de todos los clichés de la poesía amorosa medieval introduce una voz que el poeta contradice. Las convenciones del amor cortés eran compartidas por el contexto de cultura del poeta y sus lectores comprendían la contradicción con la dama oscura. Los lectores actuales logran también comprender esa voz debido a la forma de atribución utilizada por el autor. Al decir que los ojos de su amada no son para nada como el sol, queda implícito que "alguien" ha dicho que los ojos de "su" amada son como el sol. De la misma forma, si los labios de la dama oscura no son más rojos que el coral, se alude implícitamente a una voz que definió los labios rojos como el coral. Más adelante, las oraciones condicionales se dirigen específicamente a aquellos que sostienen que los cabellos amados deben ser de una forma en especial y que la blancura de los senos es un rasgo de la amada ideal. La afirmación producida por el yo lírico acerca de haber visto rosas pero que estas no están en las mejillas de la dama incorpora otra voz que dice que "su" dama tiene rosas en las mejillas. El mismo tipo de atribución se presenta en la línea que niega haber visto a una diosa caminar, donde está implícito que otra voz comparó el andar de su dama con el de una diosa. En todos estos casos, la polaridad negativa introduce otras voces que discuten con el poeta.

La heteroglosia del soneto permite crear una dama más terrenal, ridiculizar los valores atribuidos a las damas de otros poemas y ensalzar, a su vez, los valores más terrenales de esta dama oscura. Es muy interesante observar que allí donde el contexto de cultura del poeta no es compartido por el lector, el 
soneto provee ese contexto para permitir la comprensión de la lectura aún para un lector actual. Ese puente de comprensión entre el contexto de producción en el siglo XVI y la actualidad es construido por la atribución de voces y la distribución de los significados interpersonales que indican al lector cuál es la posición del yo lírico frente a esas otras voces.

El soneto analizado alinea al lector en los valores que el poema defiende o ataca a través de la negociación de actitudes que logran construir un mismo sentido que se puede comprender tanto en el siglo XVI como en el siglo XXI.

Si, como vimos en la cita de White (2004), los significados interpersonales tienen el poder de influir en la "naturalización de actitudes, creencias y supuestos, de manera más indirecta, más implícita", entonces podemos suponer que los sonetos a la dama oscura han contribuido a naturalizar actitudes acerca del amor que se trasladaron a varias generaciones y contextos de cultura. 


\section{Referencias bibliográficas}

Bloom, H. (2001). Shakespeare: la invención de lo humano. Bogotá: Editorial Norma.

---. (2005). Genios. Un mosaico de cien mentes creativas y ejemplares. Bogotá: Editorial Norma.

---. (2011). Anatomía de la influencia. Buenos Aires: Taurus.

Bowers, R. (2007). "Reviewed Work: The Cambridge Companion to Shakespeare's Poetry by Patrick G. Cheney" Renaissance Quarterly, 60 (4), 1461-1463.

Bruner, J. (1990). Acts of Meaning. Harvard: Harvard University Press.

Cureton, R. (2000). "Jakobson Revisited: Poetics, Subjectivity, and Temporality”. Journal of English Linguistics, 28.

Ducrot, O. (1988). "Argumentación y 'topoi' argumentativos". Beatriz Lavandera (Ed.) Lenguaje en contexto, 63-84.

---. (2004). "Sentido y argumentación". Elvira de Arnoux y María Marta García Negroni (Comp.) Homenaje a Oswald Ducrot. Buenos Aires: Eudeba, 370-395.

Eggins, S. and J. Martin (1994). An Introduction to Systemic Functional Linguistics. London: Pinter.

---. (1997). "Genres and registers of discourse". Teun van Dijk (Ed.) Discourse as Structure and Process. London: Sage, 230-256.

Ellrodt, R. (1997). "Shakespeare the non-dramatic poet". Stanley Wells (Ed.) The Cambridge Companion to Shakespeare Studies. Cambridge: CUP.

French, M. (1981). Shakespeare's Division of Experience. New York: Summit Books.

Fuller, J. (1972). The Sonnet. London: Methuen. 
Ghio, E. y Fernández, M.D. (2008). Lingüística Sistémico Funcional. Aplicaciones a la lengua española. Santa Fe: UNL.

Gray, H. D. (1917). "Shakespeare's Last Sonnets". Modern Language Notes, 32 (1), 17-21.

Halliday, M.A.K, (1994). An Introduction to Functional Grammar. New York: Arnold.

---. (1997 [1994]). An Introduction to Functional Grammar (2a ed.). London: Arnold. Harrison, C. and Wood. P. (Ed.)

--- (2003). Art in Theory 1900-2000. London: Blackwell Publishing.

Jakobson, R. (1985). Ensayos de Lingüística General. Barcelona: Planeta Agostini.

---. (2006). "El arte verbal en 'Th'expence of Spirit' de Shakespeare”. Ensayos de Poética. México: Fondo de Cultura Económica.

Jitrik, N. (2008). La memoria compartida. Buenos Aires: Centro Editor de América Latina.

Keach, W. (1977). "Reviews: Elizabethan Erotic Narratives: Irony and Pathos in the Ovidian Poetry of Shakespeare, Marlowe and Their Contemporaries" Journal of European Studies, 1981: 11, 54. Rutgers, N. J: Rutgers University Press, and Hassocks, London: Harvester Press.

Martin, J. and Rose, D. (2003). Working with Discourse. London: Continuum.

Martin, J.R. y White P.R.R. (2005). The Language of Evaluation. Hampshire: Palgrave Macmillan.

Rosmarin, A. (1985). "Hermeneutics versus Erotics: Shakespeare's Sonnets and Interpretive History". PMLA, 100 (1), 20-37. 
Shakespeare, W. (1978). Shakespeare's sonnets, Edited with analytic commentary by Stephen Booth. Massachusetts: Yale University.

---. (2009). Sonetos y Lamento de una amante. Buenos Aires: Paradiso. (Trad. A. Ehrenhaus).

Tynianov, I. (1995). "Ritmo como factor constructivo del verso". Emil Volek (Comp.) Antología del Formalismo Ruso y el grupo Bajtín. Madrid: Fundamentos.

White, P.R.R. (2001) "Introduction" Appraisal. An Overview. https://www.grammatics.com/appraisal/appraisalguide/unfr amed/appraisal-overview.htm

Fecha de recepción: 30/05/19

Fecha de aceptación: 22/10/19 